\title{
NUMERICAL SIMULATION FOR CONVECTION OF DECONTAMINATION AGENT IN A CLOSED ROOM
}

\author{
H. Hayakawa ${ }^{1}$, M. Suzuki ${ }^{2}$, M. Yamamoto ${ }^{3}$, M. Kawai ${ }^{4}$ and K. Sakamoto ${ }^{5}$, \\ ${ }^{1}$ Graduate School of Mechanical Engineering, Tokyo University of Science \\ ${ }^{2}$ Clean Engine Team, Aviation Program Group, Japan Aerospace Exploration Agency \\ ${ }^{3}$ Department of Mechanical Engineering, Tokyo University of Science \\ (yamamoto@rs.kagu.tus.ac.jp) \\ ${ }^{4}$ Numerical Engineering Dept, IHI \\ ${ }^{5}$ Defense Project Collaboration Div, IHI
}

\begin{abstract}
Recently, terrorism is one of most daunted dangers in the world. Various terrorisms can come about. Biological and chemical terrors have particularly high mortality rate, and tend to wreak the secondary disaster. For these reasons, research and development of measures for decontaminations are emergent and of key issue on a world scale. One of these requirement is gas decontamination technique. In the system of gas decontamination, the gaseous decontamination agent is supplied to a contamination surface. The gas decontamination does not cause corrosion and rust to the target and has high applicability to non-water-resistant targets. However, the research and development of decontamination technique are of great difficulty, and the experiments are highly risky and also legallyprohibited in many cases. Therefore, in this study, computational fluid dynamics is used to simulate the flow and concentration fields where gaseous decontamination flows in a room which is enclosed by contaminated walls. We investigate the local concentrations of decontamination agent, and find that the introduction of circulators is effective to get the uniform concentration of decontamination agent in the room. Finally, the optimum arrangement of the circulators is proposed.
\end{abstract}

Keywords: Computational Fluid Dynamics, Decontamination, Internal Flow, Mass Transfer 


\section{INTRODUCTION}

There have been various terror attacks around the world since 9.11 in 2001 [1]. Terrorists hold extremely wide-ranging means of terrors such as bombs and poison gases. Above all, nuclear and biochemical weapons have particularly high mortality rate so that they are recognized as being the most dangerous in all other ways of terror. Among these weapons, biochemical weapons are relatively-easy to be produced so that they are at high risk of being used in terrorisms. Therefore, research and development of measures and decontamination for biochemical weapons are emergent.

Decontamination methods are classified into two general groups, physical decontamination and chemical decontamination [2]. The former represents a decontamination method that directly remove contamination matter from contaminated objects due to wipe or washup. Although this method is easy and cheap, it cannot be applied to nicety and complicated shaped objects like a PC. In addition, complete removal of contamination is difficult for a physical decontamination method. In contrast, the latter uses chemical reactions to decontaminate so that it can be applied to precision equipments including a PC. Additionally, the waste substances do not hold toxicity. Chemical decontamination is classified further detailed groups, liquid decontamination and gas decontamination. Today, the chemical decontamination using liquid is the most popular way of decontamination. However, it cannot be applied to non-water resistant targets like electronic devices. On the other hand, gas decontamination does not cause corrosion and rust to the target, and has high applicability to non-waterresistant targets [3]. However, the research and development of decontamination technique are of great difficulty since the experiments are highly risky and are legally-prohibited in many cases. Moreover, in gas decontamination technique, colorless gas is used so that it is particularly difficult to measure. Therefore, numerical simulation is useful to research it.

In this study, computational fluid dynamics is used to simulate the flow and concentration fields where gaseous decontamination flows in a room which is enclosed by contaminated walls. We investigate the effect of circulators on concentration fields and propose the optimum arrangement of the circulators.

\section{NUMERICAL PROCEDURE}

The flow and concentration fields with decontamination agent in a room shown in Figure 1 is computed in this study. Since the flow is assumed to be multiphase flow consisting of air and decontamination agent, we use a homogenous fluid model. As the treatment of multiphase flow, we apply the two-way coupling method, that is, the concentration and the flow affects each other. The numerical procedures for the flow field and the concentration field will be described below. 


\subsection{Flow field}

It is assumed that the flow field is three-dimensional and incompressible. We use the conservation equations of mass and momentum expressed by Eqs. (1) and (2) as the governing equations. For pressure-velocity coupling, the Low-Mach-Number approximated MAC method (marker and cell method) is used. Since we treat two-phase flow consisting of air and decontamination agent, the mixture density calculated by Eqs. (3) and (4) at each grid points are considered in the flow calculations. No-slip condition between air and decontaminant agent is imposed to calculate the mixture gas velocity, $\vec{u}_{m i x}$.

$$
\begin{gathered}
\frac{\partial \rho_{\text {mix }}}{\partial t}+\nabla \cdot\left(\rho_{\text {mix }} \vec{u}_{\text {mix }}\right)=0 . \\
\frac{\partial \rho_{m i x} \vec{u}_{m i x}}{\partial t}+\vec{u}_{\text {mix }} \cdot \nabla\left(\rho_{\text {mix }} \vec{u}_{m i x}\right)=-\nabla p+\mu_{\text {air }} \nabla^{2} \vec{u}_{m i x}-\left(\rho_{s}-\rho_{\text {mix }}\right) \vec{g} . \\
\rho_{\text {mix }}=\alpha_{\text {air }} \rho_{\text {air }}+\alpha_{d} \rho_{d} . \\
\alpha_{\text {air }}+\alpha_{d}=1 .
\end{gathered}
$$

In this above equations, $\alpha$ is the volume fraction, $\rho$ is the density, $u$ is the flow velocity, $t$ is the time, $p$ is the pressure, $\mu$ is the viscosity coefficient, $g$ is the gravity acceleration, index air is the air, $d$ is the decontamination agent, mix is the mixture gas and $s$ is the average value of overall computational domain. Since the volume fraction of decontaminant agent is low enough, the viscosity of air is used in Eq. (2). The gravity term is calculated by the difference between the average density and the local density.

A finite difference method is applied to discretize the governing equations. The Kawamura-Kuwahara [4] third-order upwind scheme is used for the convection terms, the Euler explicit scheme for the time marching terms, and the second-order central difference scheme for the other terms.

\subsection{Concentration field}

For the concentration field, the governing equation is given as

$$
\frac{\partial C_{d}}{\partial t}+\vec{u}_{d} \cdot \nabla C_{d}=D_{d} \nabla^{2} C_{d}
$$

where $C$ is mol concentration and $D$ is the diffusion coefficient. $D$ is calculated from Chapman-Enskog's equation [5]. The numerical difference schemes are employed in similar to the flow field. 


\section{COMPUTATIONAL CONDITION}

Computations are performed for the flow in a simplified room as shown in Figure 1. The decontaminant generator is installed at the center-bottom of the room. Fans are installed in six ways (i.e. Case0 to Case5) as shown in Figure 1(a). There is no fan in Case0, one fan in Case 1 to Case 3 and two fans in Case 4 and Case5. The number of grid points is $150 \times 100 \times$ 70 , clustered near the decontaminant generator, walls, inlet boundary and outlet boundary, where the gradients of physical quantities are expected to be steep. Decontamination agent is considered to be ozone and be supplied into the room during a specified time interval. On the wall, no-slip boundary condition is applied for the velocity, and first order extrapolation is applied for the others. At the inlet boundary, velocity and concentration of ozone is fixed and others are extrapolated from the computational domain. At the outlet boundary, velocity is fixed and others are extrapolated. As the treatment of fan's effect, velocity in $x$ direction is fixed in front of the fan.

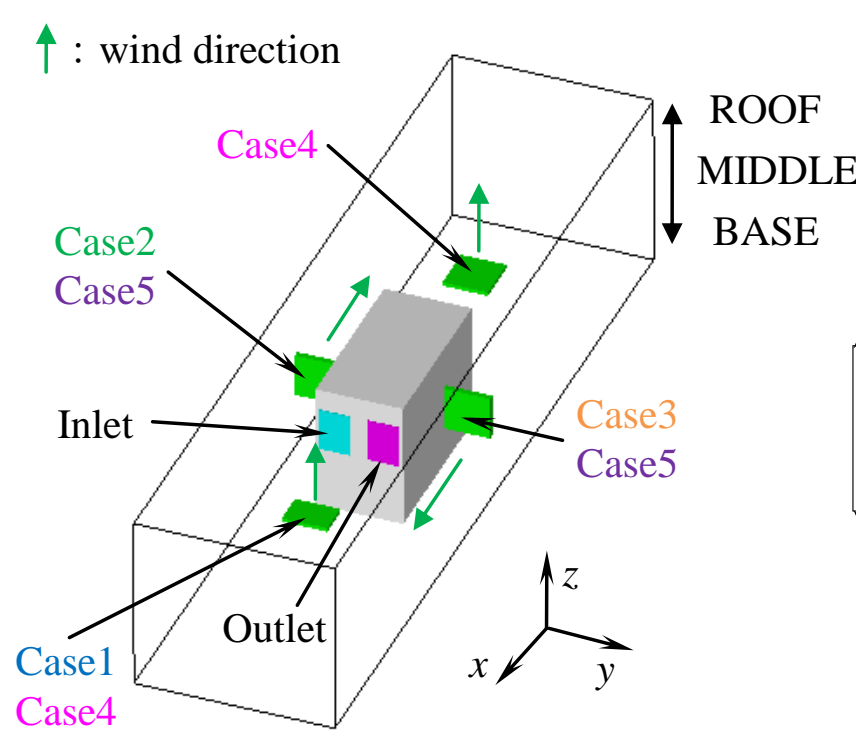

(a) Installation conditions

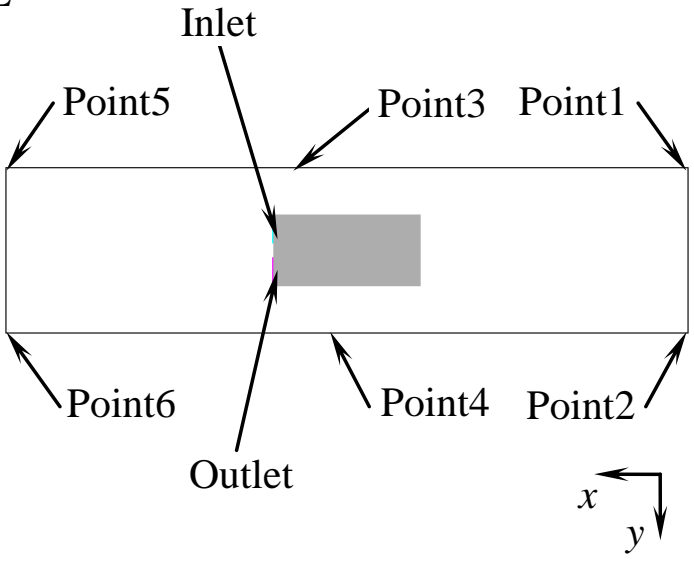

(b) Measurement points

Figure 1 Installation conditions and measurement points

\section{NUMERICAL RESULTS AND DISCUSSION}

\subsection{Effect of fan}

Figure 2 denotes the distribution of ozone concentration in Case0 (i.e. the non-fan condition). In this figure, $t$ and the concentration of ozone is normalized by the maximum time of ozone supply and supplied concentration of ozone, respectively. The molecular mass of ozone is heavier than that of air so that ozone settles at the bottom of the room in the nonfan condition. Therefore, the concentration gets higher at the bottom and gets lower at the roof. Installing fans like Case1 to Case5, air and ozone are mixed up and leads uniformity of 
ozone concentration. The behavior of ozone in Case2 is shown in Figure 3. In this figure, it is observed that ozone is accelerated in $x$ direction and it crashes at the forward wall and rolls up to the roof. This trend is also observed in other cases where a fan is installed facing to the $x$ direction.

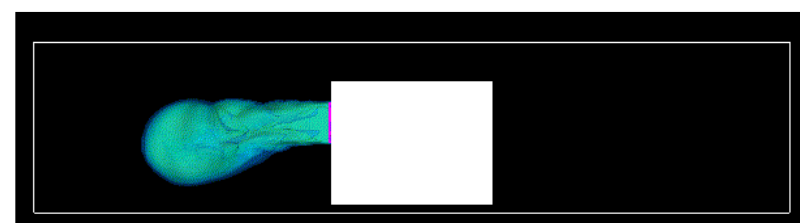

(a) $t=0.03$

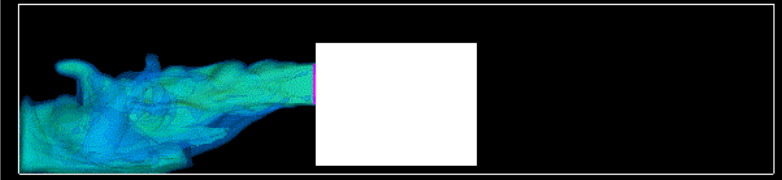

(b) $t=0.06$

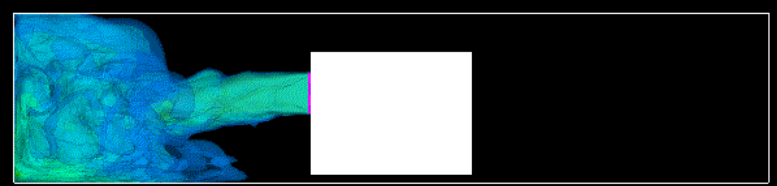

(c) $t=0.11$

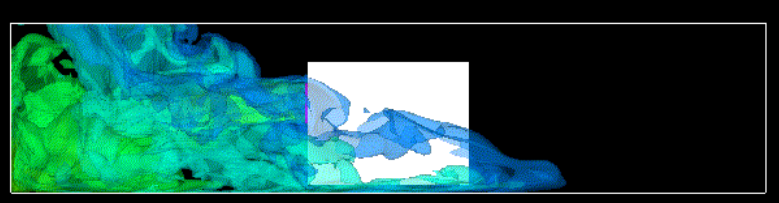

(d) $t=0.26$

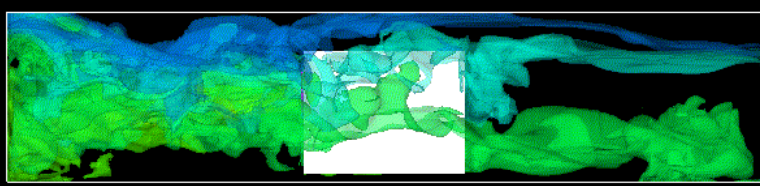

(e) $t=0.86$

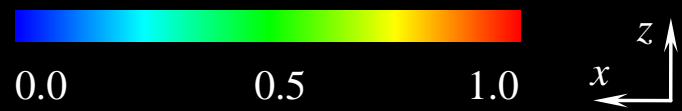

Figure 2 Concentration of ozone (Case 0$)$

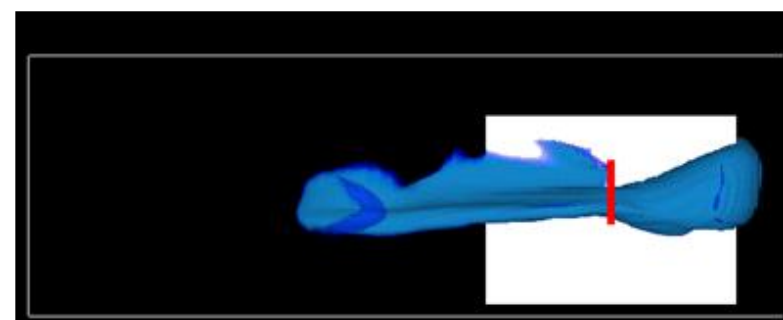

(a) $t=0.0024$

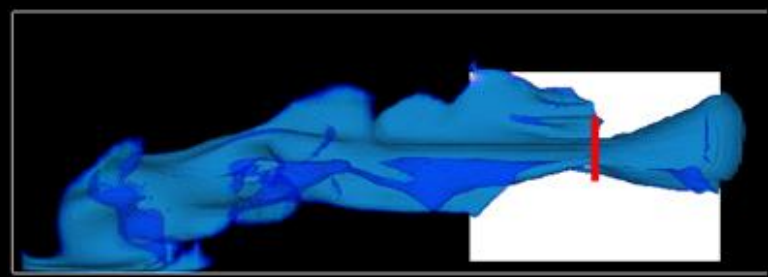

(b) $t=0.0034$

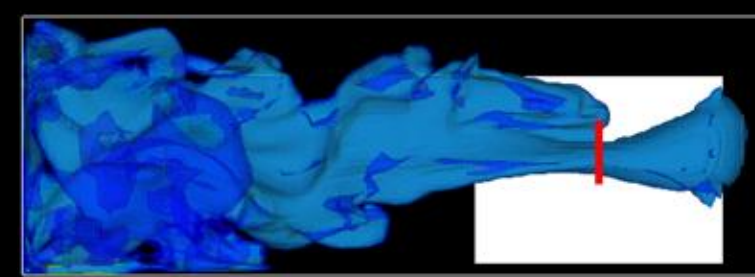

(c) $t=0.0044$

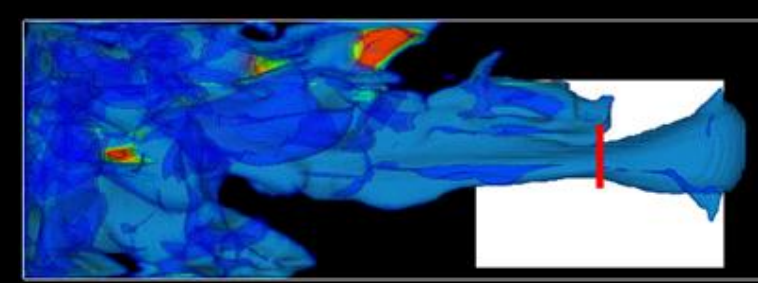

(d) $t=0.0054$
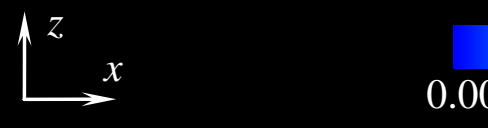

0.01

0.02

Figure 3 Distribution of ozone concentration (Case2) 


\subsection{Evaluation criteria for each case}

We adopt six points illustrated in Figure 1(b) (i.e. Point1 to Point6) at each three surfaces shown in Figure 1(a) (i.e. BASE, MIDDLE and ROOF) to measure the local concentration. Hence, concentration of ozone is investigated at these eighteen points. The optimum installation of fans is decided by two evaluation criteria as follows,

(a) The average concentration of ozone in the room

(b) Differences among concentrations of ozone at the measurement points

The case is evaluated as optimum where the value (a) is high and the value (b) is low.

\subsection{Evaluation based on criterion (a)}

Figure 4 denotes the temporal change of the average concentration of ozone for each case. In this figure, Case 4 is the highest in the concentration of ozone followed by Case 5. The amount of inflow ozone is same in all cases so that the average concentration of ozone depends only on the amount of outflow ozone. In other words, criterion (a) evaluates how much ozone is kept in the room. The average concentration of ozone in Case 3 is the lowest in all cases. This is because ozone is pushed to one side of the room and dense ozone is gathered near the outlet boundary, and thus much ozone is sucked out from the outlet. Figure 5 compares the distributions of ozone at the MIDDLE plane in Case3 and Case5. In Figure 5(a) (i.e. Case3), ozone is pushed to the left region and leads high concentration of ozone near the outlet boundary. In Figure 5(b) (i.e. Case5), installed two fans face to opposite directions so that ozone is pushed to both directions, left and right. In this way, ozone disperses widely and uniformly in the room.

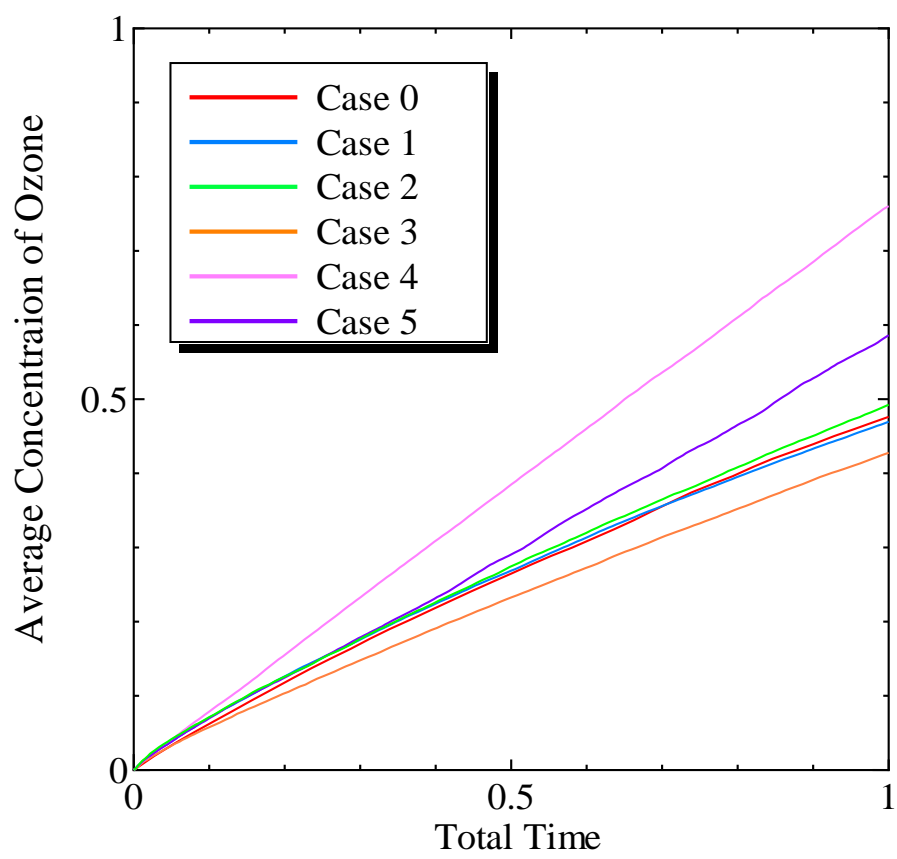

Figure 4 Average concentration of ozone 


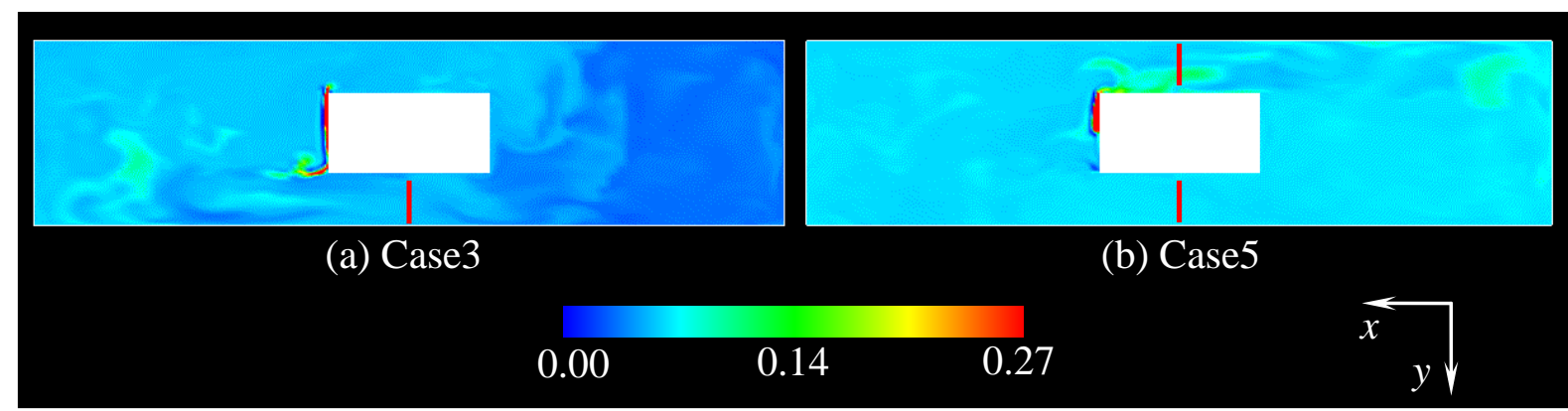

Figure 5 Concentration of ozone $(t=0.086)$

\subsection{Evaluation based on criterion (b)}

We define the saturation time that difference between average concentration and all eighteen local concentrations falls below ten percents of the average concentration (i.e. Eq. (6)),

$$
10 \geq \frac{C_{i}-C_{a v e}}{C_{\text {ave }}} \times 100
$$

where $C_{i}$ is the local concentration of ozone at each eighteen measurement points and $C_{a v e}$ is the average ozone concentration of overall computational domain.

Table 1 denotes the saturation times of each case. The saturation time is the shortest in Case 5 and Case 4 follows it. In Case0, the non-fan condition, ozone concentration does not saturate even after the maximum ozone supply time so that it can be said that a fan is considerably effective to uniformize the concentration. The biggest factor in the non-uniformity in Case 0 is the difference in concentration of ozone between ROOF and Base. On the other hand, in Case1 to Case5, dilution of ozone at the ROOF is relieved by the effect of fans. In Case1 and Case4, fans raise ozone upwards and supply it to the ROOF. In Case2, Case 3 and Case5, ozone rolls up to the ROOF after impacting to the wall as shown in Figure 3.

Table 1 Saturation time

\begin{tabular}{|c|c|c|c|c|c|}
\hline Case0 & Case1 & Case2 & Case3 & Case4 & Case5 \\
\hline- & 0.93 & 0.54 & 0.82 & 0.49 & 0.34 \\
\hline
\end{tabular}

\subsection{Flow pattern of optimum case}

Case 4 and Case 5 demonstrate the favorable results based on both criteria (a) and (b). Figure 6 shows the stream line. Case 4 and Case 5 are similar in having a flow pattern that flows along the side walls of the room avoiding outlet boundary located at the center of the room. Figure 6 shows that both Case 4 and Case 5 have a large circulating structure covering the entire region of the room. 
Figures 7 and 8 exhibit the distributions of ozone in Case4 and Case5, respectively. In both figures, the distributions of ozone in Case 4 and Case 5 are shown together with that of Case0. It should be noted that $x-z$ section is shown in Figure 7 and $x-y$ section is shown in Figure 8. In both Case4 and Case5, ozone disperses across the room rapidly comparing to Case0. Considering this fact with stream line shown in Figure 6, it is thought that ozone keeps up a stream circulating along the side walls avoiding the outlet boundary, and it contributes to the elevation of average concentration of ozone.

In this study, the situation is assumed that walls are polluted so that high concentration near the walls is required. Additionally, aiming for high average concentration of ozone in the room, outlet boundary should be settled away from ozone. Therefore, decontaminant generator with outlet boundary should be installed at the center of the room and fans should be installed so as to make ozone flows near the walls.

\section{CONCLUSION}

In this study, we performed the simulations for convection and diffusion of decontamination agent in a closed room. The obtained remarks are summarized as follows:

- In non-fan case, ozone tends to settle down to the bottom of the room, because of the large density.

- Installation of fans is considerably effective in uniformizing the concentration of decontamination agent.

- Case4 and Case5 are regarded as the optimum arrangement, based on the criteria (a) and (b).

- In Case4 and Case5, ozone keeps up the stream circulating along the side walls and disperses effectively in the entire room.

\section{REFERENCES}

[1] Integrated Terrorism Assessment Centre., "Terrorist Incidents Since 9/11," http://itac.gc.ca/thrt/ncdnts-eng.asp (accessed 2012-04-20)

[2] Seto Y., "Decontamination of Chemical and Biological Warfare Agents," YAKUGAKU ZASSHI., 129(1), 53-69, 2009.

[3] Ministry of Defense Technical Research \& Development Institute., "Defense Technology Symposium 2010" http://www.mod.go.jp/trdi/research/dts2010.files/R2/R25.pdf (accessed 2012-4-20)

[4] Kawamura T., Kuwahara K., "Computation of High Reynolds Number Flow around a Circular Cylinder with Surface Roughness,” AIAA-84-0340, 1984.

[5] Kukkonen J., Riikonen K., Nikmo J., Jappinen A., Nieminen K., "Modeling Aerosol Processes Related to the Atmospheric Dispersion of Sarin," Int. J. of Hazardous Materials., A85, 165-179, 2001. 


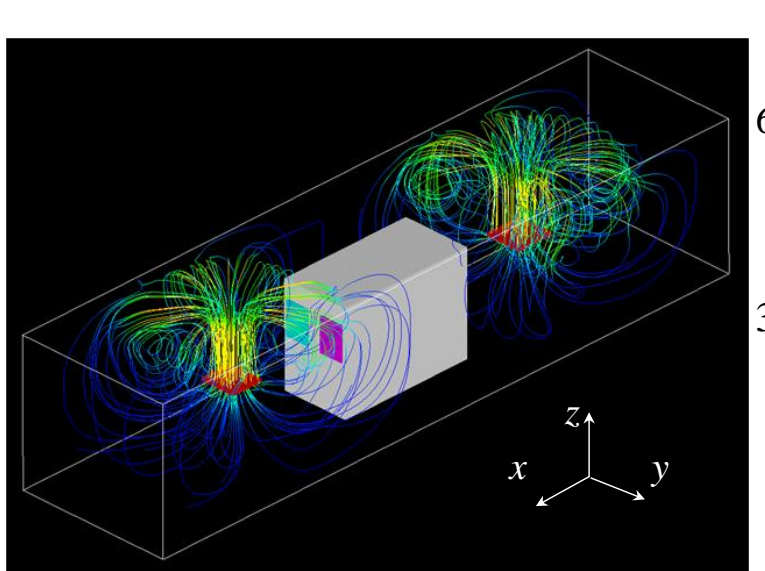

(a) Case4

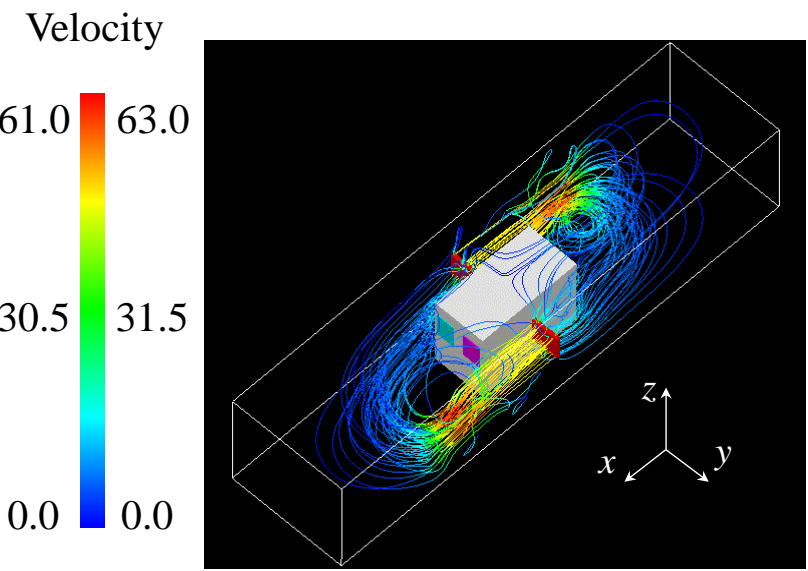

(b) Case5

Figure 6 Stream line in optimum cases

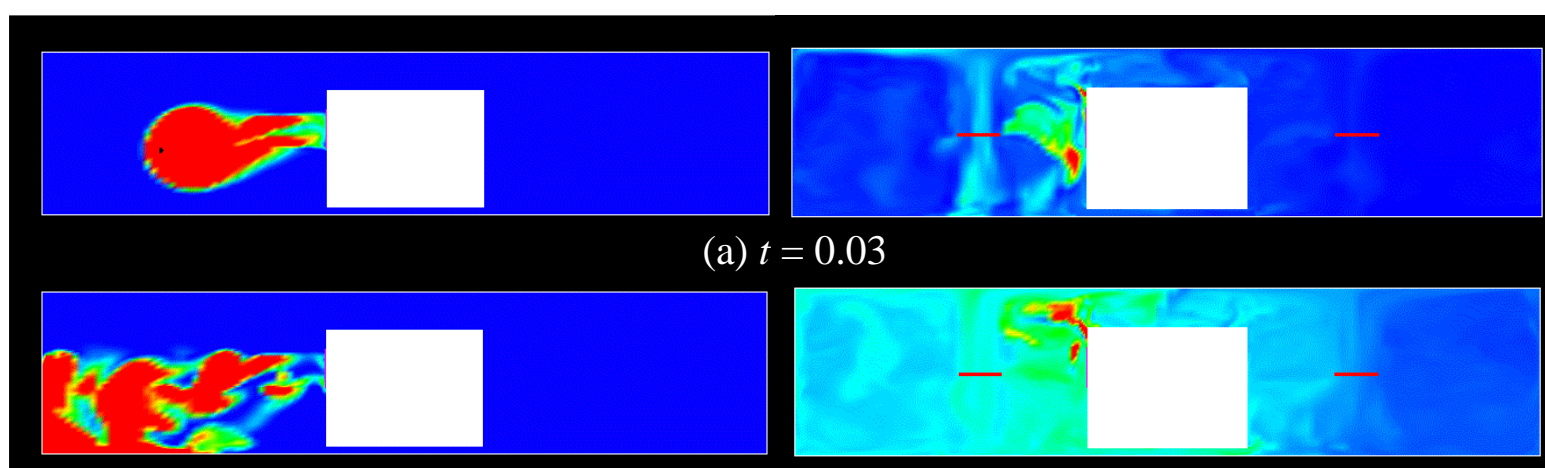

(b) $t=0.07$

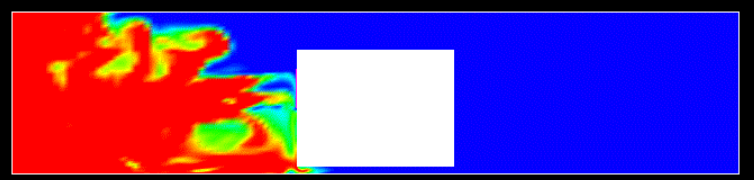

(c) $t=0.21$
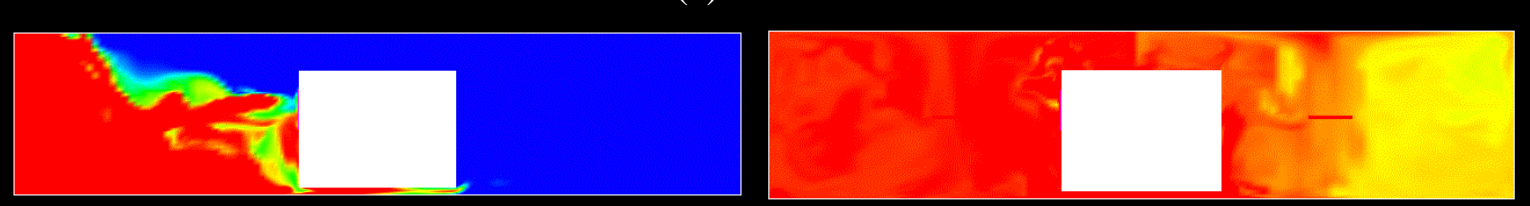

(d) $t=0.29$

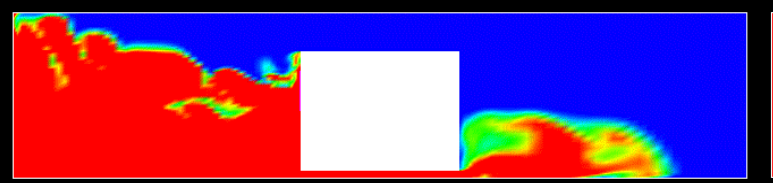

(e) $t=0.36$

(a) $\mathrm{Case} 0$
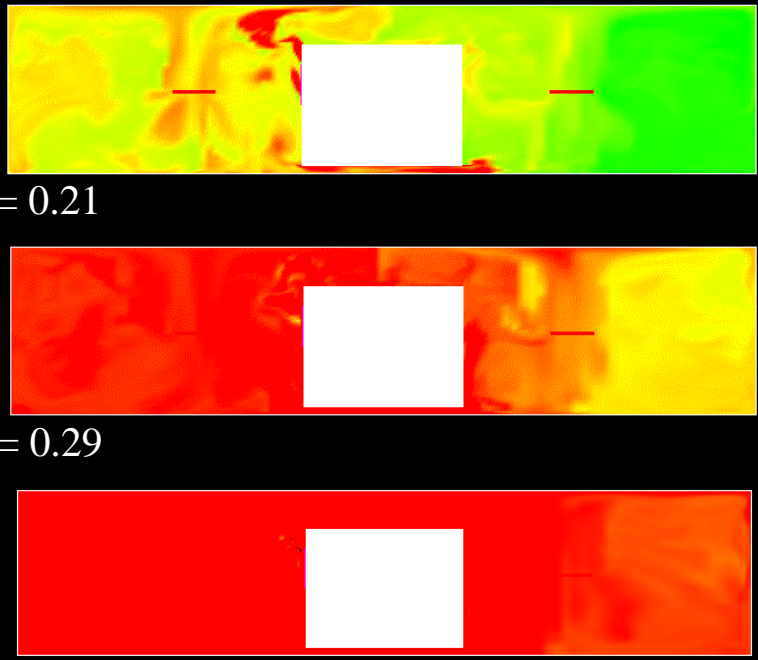
(b) Case 4

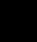

0.2

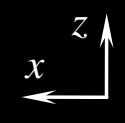

Figure 7 Temporal change of distribution of ozone concentration (Case4) 


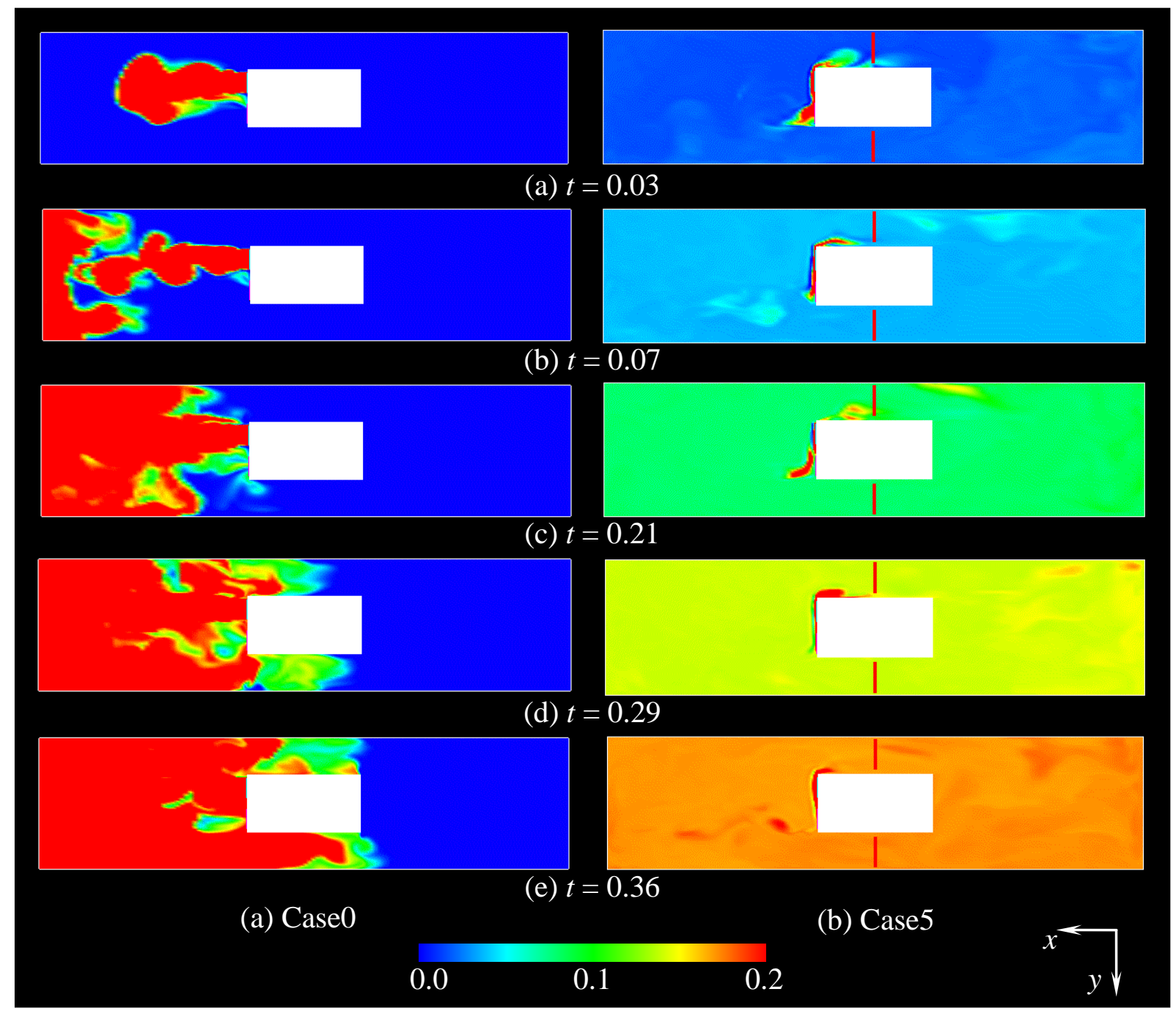

Figure 8 Temporal change of distribution of ozone concentration (Case5) 